\title{
INTERSECTION PATTERNS OF CURVES
}

\author{
JACOB FOX, JÁNOS PACH, AND CSABA D. TÓTH
}

\begin{abstract}
We prove that for every $k \in \mathbb{N}$ there is a constant $c_{k}>0$ with the following property. Every set of $n>1$ continuous curves in the plane, any pair of which intersect in at most $k$ points, has two disjoint subsets $A$ and $B$, each of size at least $c_{k} n$, such that either every curve in $A$ intersects all curves in $B$, or no curve in $A$ intersects any curve in $B$. This statement does not remain true if we drop the condition on the number of intersection points per pair.
\end{abstract}

\section{Introduction}

A classical result of Erdős and Szekeres $[\mathbf{1 0}]$ in Ramsey theory states that every graph on $n$ vertices contains a clique or an independent set of $\operatorname{size}^{\dagger}$ at least $\frac{1}{2} \log n$. This bound is tight up to a constant factor: Erdös [7] showed that there exists a graph on $n$ vertices, for every integer $n>1$, with no clique or independent of more than $2 \log n$ vertices. Erdös and Hajnal [8] proved that certain graphs contain much larger cliques or independent sets: For every hereditary family $\mathcal{F}$ of graphs other than the family of all graphs, there is a constant $c(\mathcal{F})>0$ such that every graph in $\mathcal{F}$ on $n$ vertices contains a clique or an independent set of size at least $e^{c(\mathcal{F}) \sqrt{\log n}}$. (A family of graphs is hereditary if it is closed under taking induced subgraphs.) They also asked whether this bound can be improved to $n^{c(\mathcal{F})}$. This motivates the following definitions.

Definition 1. A family $\mathcal{F}$ of finite graphs has the Erdös-Hajnal property if there is a constant $c(\mathcal{F})>0$ such that every graph in $\mathcal{F}$ on $n$ vertices contains a clique or an independent set of size $n^{c(\mathcal{F})}$.

A family $\mathcal{F}$ of finite graphs has the strong Erdös-Hajnal property if there is a constant $b(\mathcal{F})>0$ such that for every graph $G$ in $\mathcal{F}$ on $n$ vertices, $G$ or its complement $\bar{G}$ contains a balanced complete bipartite graph of size $b(\mathcal{F}) n$.

A complete bipartite graph is said to be balanced if its vertex classes differ in size by at most one. A balanced complete bipartite graph with $n$ vertices is called a bi-clique of size $n$.

Alon et al. [2] proved that if a hereditary family of graphs has the strong Erdős-Hajnal property, then it also has the Erdős-Hajnal property. For partial results on the Erdös-Hajnal problem, see $[\mathbf{3}],[\mathbf{4}],[\mathbf{5}]$, and $[\mathbf{9}]$.

Given a family $\mathcal{S}$ of subsets of $\mathbb{R}^{d}$, its intersection graph $G_{\mathcal{S}}$ is defined as the graph on the vertex set $\mathcal{S}$, whose two vertices $S, T \in \mathcal{S}$ are connected by an edge if and only if $S \cap T \neq \emptyset$. As noted by Ehrlich, Even, and Tarjan [6], not all graphs can be realized as intersection graphs of connected sets in the plane. For instance, the bipartite graph on 15 vertices formed by replacing each edge of $K_{5}$ by a path of length 2 has no such realization. This implies, using the above

2000 Mathematics Subject Classification 05D10, 05C62 (primary), 05C35, 06A07 (secondary).

The first author is supported by an NSF Graduate Research Fellowship and a Princeton Centennial Fellowship. The second author is supported by NSF Grant CCF-05-14079, and by grants from NSA, PSC-CUNY, the Hungarian Research Foundation OTKA, and BSF.

$\dagger$ All logarithms in this paper are of base two. 
result of Erdös and Hajnal, that the intersection graph of any $n$ connected sets in the plane contains a clique or an independent set of size $e^{c \sqrt{\log n}}$, for some absolute constant $c>0$. This general bound has been improved for intersection graphs of certain geometric objects. Pach and Solymosi [18] proved that the family of intersection graphs of line segments in the plane has the strong Erdős-Hajnal property. Later, Alon et al. [2] generalized this result and showed that the intersection graphs of semialgebraic sets of constant description complexity in $\mathbb{R}^{d}$ also have the strong Erdős-Hajnal property. Recently, Basu [4] has further extended this result to a broader class of algebraically defined sets.

A (simple) curve in the plane is the range of a continuous (bijective) function $f: I \rightarrow \mathbb{R}^{2}$ whose domain is a closed interval $I \subset \mathbb{R}$. A collection of curves is $k$-intersecting if any two of them intersect in at most $k$ points. A 1-intersecting collection of curves is also called a family of pseudo-segments. A collection of portions of algebraic curves of maximum degree $d$ in general position is $d^{2}$-intersecting. Clearly, the intersection graphs of $k$-intersecting collections of curves form a hereditary family. The main result of this paper is the following.

THEOREm 1.1. Let $C$ be a $k$-intersecting collection of $n>1$ curves in the plane such that the number of intersecting pairs of curves is at least $\varepsilon n^{2}$, for some $\varepsilon>0$. Then the intersection graph of $C$ contains a bi-clique of size at least $c_{k} \varepsilon^{c} n$, where $c$ is an absolute constant and $c_{k}>0$ depends only on $k$.

The constant $c$ in Theorem 1.1 can be taken to be 64 , but probably this value can be much reduced.

A separator for a graph $G=(V, E)$ is a subset $V_{0} \subset V$ such that there is a partition $V=V_{0} \cup V_{1} \cup V_{2}$ with $\left|V_{1}\right|,\left|V_{2}\right| \leq \frac{2}{3}|V|$ and no vertex in $V_{1}$ is adjacent to any vertex in $V_{2}$. According to the Lipton-Tarjan separator theorem [17], every planar graph with $n$ vertices has a separator of size $O(\sqrt{n})$. Fox and Pach [14] proved the following generalization of this result: The intersection graph of any finite collection of curves in the plane with a total of $m$ intersection points (crossings) among them has a separator of size $O(\sqrt{m})$.

Suppose now that $C$ is a $k$-intersecting collection of $n>1$ curves in the plane that does not satisfy the condition in Theorem 1.1, for some $\varepsilon>0$. That is, $C$ has fewer than $\varepsilon n^{2}$ intersecting pairs of curves. If $\varepsilon=\varepsilon_{k}$ is sufficiently small, then we can apply the above mentioned separator theorem from [14] with $m<k \varepsilon n^{2}$ to conclude that the complement of the intersection graph of $C$ contains a bi-clique of size at least constant times $n$. Therefore, Theorem 1.1 implies

Corollary 1.2. For every $k \in \mathbb{N}$, the family of intersection graphs of $k$-intersecting collections of curves in the plane has the strong Erdös-Hajnal property. That is, for every $k \in \mathbb{N}$, there is a constant $c_{k}>0$ such that the intersection graph of every $k$-intersecting collection of $n$ curves or its complement contains a bi-clique of size $c_{k} n$.

The last statement settles a problem raised in [19].

Note that Corollary 1.2 is false if we drop the condition that the number of intersection points between two curves is bounded by a constant. Indeed, combining a result of Fox [11] with the fact that the incomparability graph of any partially ordered set can be obtained as the intersection graph of a collection of curves $[\mathbf{1 9}, \mathbf{2 0}]$, we can conclude that for every $\varepsilon>0$, there is a collection of $n$ curves in the plane whose intersection graph $G$ contains no bi-clique of size $\frac{14 n}{\varepsilon \log n}$, and $\bar{G}$ contains no bi-clique of size $n^{\varepsilon}$.

A Jordan region is a subset of the plane that is homeomorphic to a closed disk. We say that a Jordan region $R$ contains another Jordan region $S$ if $S$ lies in the interior of $R$. Clearly, if two Jordan regions intersect, then either their boundary curves intersect or one contains the other in its interior. A crossing between a pair of Jordan regions is defined as an intersection point 
of their boundaries or a containment between them. (Two Jordan regions may have arbitrarily many crossings.)

Corollary 1.2 can now be generalized as follows.

TheOREM 1.3. Let $k \in \mathbb{N}$, and let $\mathcal{R}$ be any family of Jordan regions whose boundaries form a $k$-intersecting family of curves. Let $\mathcal{R}_{k}$ denote the family of all sets that can be obtained by taking the union of at most $k$ elements of $\mathcal{R}$. Then the family of intersection graphs of all finite collections of elements of $\mathcal{R}_{K}$ has the strong Erdös-Hajnal property.

Alon et al. $[\mathbf{2}]$ proved that the intersection graphs of collections of semi-algebraic sets of constant description complexity in $\mathbb{R}^{d}$ have the strong Erdös-Hajnal property. Theorem 1.3 generalizes the planar case of this result. Indeed, the boundary of a semi-algebraic set of bounded description complexity in the plane is the union of a bounded number of algebraic curves of bounded degree, any two of which either intersect in a bounded number of points or overlap. By slightly perturbing the semi-algebraic sets, while maintaining their intersection pattern and their description complexity, we can assume that their boundaries do not overlap. By slightly "fattening" these sets, if necessary, we can also assume that each of them is the union of a constant number of Jordan regions.

The paper is organized as follows. Sections $2-5$ add up to the proof of Theorem 1.1. Given a $k$-intersecting collection of curves, it is enough to find a large bi-clique in the intersection graph of some portions of the curves. If we truncate the curves at their intersection points with one or two special curves (called grounds), we obtain a set of grounded or double grounded curves (defined below). The arrangement of curves that connect points on two grounds is isomorphic to an arrangement of curves connecting points on two parallel lines, $x=0$ and $x=1$, which we call $[0,1]$-curves. Intersection graphs of $[0,1]$-curves can, in turn, be realized as incomparability graphs.

In Section 2, we prove some auxiliary results on the structure of $K_{t, t}$-free comparability and incomparability graphs. In Section 3, we introduce grounded and double-grounded collections of curves and prove a lemma on the intersection pattern of double grounded curves with other curves (Lemma 3.2). Section 4 is the heart of the proof of Theorem 1.1: we prove Theorem 1.1 for $[0,1]$-curves (Theorem 4.1). In Section 5 , we put the pieces of the puzzle together and prove Theorem 1.1.

Theorem 1.3 will be established in Section 6 . The last section contains some applications of our results as well as a few open problems.

\section{The Structure of Comparability and Incomparability Graphs}

A comparability graph of a poset $(P, \prec)$ is a graph on the vertex set $P$, where two elements of $P$ are adjacent if and only if they are comparable. The incomparability graph of a poset $(P, \prec)$ is defined analogously, except that now two elements of $P$ are connected by an edge if and only if they are incomparable.

In this section, we prove a few auxiliary results on comparability graphs and incomparability graphs of partially ordered sets (posets). The central role that partial orders play in the analysis of intersection patterns of curves can be explained by the next lemma (Lemma 2.1). It shows that in the important special case when the curves connect two parallel lines in the strip bounded by them, their intersection pattern can be fully described by a partial order.

We use the following terminology. A $[0,1]$-curve is a curve in the plane that has one endpoint on the line $L_{1}: x=0$, the other endpoint on the line $L_{2}: x=1$, and the rest of the curve is contained in the open strip between $L_{1}$ and $L_{2}$. A curve in the plane is called $x$-monotone if it intersects any vertical line in at most one point. Define a partial order $\prec$ on $[0,1]$-curves as 
follows: let $\alpha \prec \beta$ if $\alpha$ and $\beta$ are disjoint and the $y$-coordinate of $\alpha \cap L_{1}$ is smaller than the $y$ coordinate of $\beta \cap L_{1}$. For a collection $C$ of [0,1]-curves with distinct endpoints, the intersection graph is the same as the incomparability graph of the poset $(C, \prec)$. It is easy to show that every incomparability graph can be represented as the intersection graph of $x$-monotone $[0,1]$-curves.

Lemma 2.1. [20, 19] The following three statements are equivalent:

(i) $G$ is an incomparability graph.

(ii) $G$ is the intersection graph of a collection of $[0,1]$-curves.

(iii) $G$ is the intersection graph of a collection of $x$-monotone $[0,1]$-curves.

A graph is said to be $H$-free if it does not contain $H$ as a (not necessarily induced) subgraph. A graph is $r$-degenerate if each of its subgraphs contains a vertex of degree at most $r$. It is easy to see that every $r$-degenerate graph with $n>r$ vertices has at most $r n-\left(\begin{array}{c}r+1 \\ 2\end{array}\right)$ edges.

Every poset is the intersection of its linear extensions. The dimension of a poset is the minimum number of linear extensions whose intersection is that poset.

The following lemma was established in [12]; we include its very short proof for completeness.

Lemma 2.2. [12] If $G$ is a $K_{t, t}$-free incomparability graph of a 2-dimensional poset, then $G$ is $(2 t-2)$-degenerate. In particular, $G$ has at most $2 t n-\left(\begin{array}{c}2 t-1 \\ 2\end{array}\right)$ edges.

We use the notation $[n]:=\{1, \ldots, n\}$. For a permutation $\pi$ of $[n]$, let $P_{\pi}=\left([n],<_{\pi}\right)$ be the 2-dimensional partial order on $[n]$ given by $i<_{\pi} j$ if and only if $i<j$ and $\pi(i)<\pi(j)$.

Proof. Every 2-dimensional poset of $n$ vertices can be realized as $P_{\pi}$, for a suitable permutation $\pi$. Suppose for contradiction that the degree of every vertex of the incomparability graph $P_{\pi}$ is at least $2 t-1$. Notice that every $i \in[n]$ is incomparable with at most $i-1+\pi(i)-1$ other elements of $[n]$. Since each element $i \in[n]$ is incomparable with at least $2 t-1$ other elements of $P_{\pi}$, we have $\pi(i) \geq t+1$ for $i \in[t]$ and $i \geq t+1$ for $\pi(i) \in[t]$. In particular, every $i \in[t]$ is incomparable with every element $j$ with $\pi(j) \in[t]$. Hence, the incomparability graph contains $K_{t, t}$, which is a contradiction.

A partially ordered set $(P, \prec)$ is called an interval order if one can assign an interval $\left(x_{L}, x_{R}\right)$ to each $x \in P$ such that $x_{R}<y_{L}$ if and only if $x \prec y$.

Lemma 2.3. If $G$ is a $K_{t, t}$-free comparability graph of an interval order $(P, \prec)$ with $n$ elements, then $G$ has at most $2(t-1) n$ edges.

Proof. Let $P=\left\{I_{1}, \ldots, I_{n}\right\}$ be a set of $n$ intervals representing the interval order $(P, \prec)$. If there is an integer $i \in[n]$ such that at least $t$ intervals are to the left of $I_{i}$ (that is, there are at least $t$ intervals $I_{j}$ with $I_{j} \prec I_{i}$ ) and at least $t$ intervals to the right of $I_{i}$, then the comparability graph of the interval order $(P, \prec)$ contains $K_{t, t}$. So, for every $i \in[n]$, there are fewer than $t$ intervals to the left of $I_{i}$ or there are fewer than $t$ intervals to the right of $I_{i}$. Partition $P$ into two subsets $P=L \cup R$ such that $I_{i} \in L$ if and only if there are at least $t$ intervals to the left of $I_{i}$. If $L$ has at least $t$ elements, then there are at least $t$ intervals to the left of the left-most endpoint $p$ of all intervals in $L$, and all intervals of $L$ are to the right of $p$, which contradicts our assumption that $G$ is $K_{t, t}$-free. Hence, we have $|L|<t$. Since each interval in $R$ has at most $t-1$ intervals on its left, the number of edges of $G$ is at most $|L| n+(t-1)|R| \leq 2(t-1) n$.

The edge density of a graph with $n$ vertices and $m$ edges is the fraction $m /\left(\begin{array}{c}n \\ 2\end{array}\right)$ of vertex pairs connected by an edge. Next, we show that if an incomparability graph is fairly dense but 
does not contain a large bi-clique, then it has a large induced subgraph whose edge density is close to 1 .

Lemma 2.4. If $G$ is a $K_{t, t}$-free incomparability graph with $n$ vertices and $m \geq \sqrt{5 t n^{3}}$ edges, then $G$ contains an induced subgraph with at least $\frac{m^{2}}{5 n^{3}}$ vertices and edge density at least $1-10 t \frac{n^{3}}{m^{2}}$.

Proof. Let $G$ be the incomparability graph of a poset $([n], \prec)$ and assume that $i<j$ whenever $i \prec j$. Let $S \subset[n]$ be a set of integers such that $i \in S$ if and only if $i$ is incomparable with at least $\frac{m}{n}$ other elements of $[n]$. So we have $\frac{m}{n} n+n|S| \geq 2 m$, or equivalently, $|S| \geq \frac{m}{n}$.

For every $i \in S$, let $u(i)$ denote the up-degree, that is, the number of integers $j \in[n]$ such that $i<j$ and $j$ is incomparable with $i$; and similarly let $d(i)$ denote down-degree, which is the number of integers $j \in[n]$ such that $j<i$ and $j$ is incomparable with $i$. Partition $S$ into two subsets $S=U \cup D$ such that $i \in U$ if and only if $u(i) \geq d(i)$. Notice that each element of $U$ has up-degree at least $\frac{m}{2 n}$ and each element of $D$ has down-degree at least $\frac{m}{2 n}$. Since $|S| \geq \frac{m}{n}$, we have $|U| \geq \frac{m}{2 n}$ or $|D| \geq \frac{m}{2 n}$. Without loss of generality, assume $|U| \geq \frac{m}{2 n}$.

Partition $[n]$ into at most $\frac{5}{2} \frac{n^{2}}{m}$ intervals, each of length at most $\frac{m}{2 n}$. Pick one of these intervals, $I$, that contains at least $|U| /\left(\frac{5}{2} \frac{n^{2}}{m}\right) \geq \frac{m^{2}}{5 n^{3}}$ elements of $U$. We next show that the edge density of the incomparability graph of $(I \cap U, \prec)$ is close to 1 . Denote by $i$ the largest element of $I$. For every $j \in I \cap U$, let $F_{j}$ be the set of integers $f$ such that $j<f \leq i$ and $j \prec f$; and let $H_{j}$ be the set of integers $h$ such that $i<h \leq n$ and $h$ is incomparable with $j$. Notice that every element $f \in F_{j}$ is incomparable with every element $h \in H_{j}$, otherwise $j \prec f \prec h$ would contradict that every element of $H_{j}$ is incomparable with $j$. Notice, further, that $\left|F_{j}\right| \leq\left|H_{j}\right|$ since $\frac{m}{2 n} \leq u(j)=\left|H_{j}\right|+\left(i-j-\left|F_{j}\right|\right)$ and $i-j \leq|I| \leq \frac{m}{2 n}$. It follows that $\left|F_{j}\right|<t$, otherwise already the incomparability graph of $F_{j} \cup H_{j}$ would contain $K_{t, t}$. Therefore, each element $j \in I \cap U$ is incomparable with all but at most $t-1$ elements $k \in I$ with $j<k$. Hence, the edge density of the subgraph induced by $I \cap U$ is at least

$$
1-\frac{(t-1)|I \cap U|}{\left(\begin{array}{c}
|\cap \cap U| \\
2
\end{array}\right)} \geq 1-\frac{10 t n^{3}}{m^{2}} .
$$

\section{Double-Grounded Curves}

A collection $C$ of curves in the plane is grounded if there is a closed Jordan curve $\gamma$ (which is a ground for $C$ ) such that each curve in $C$ has one endpoint on $\gamma$ and the rest of the curve is in the exterior of $\gamma$. A collection $C$ of curves in the plane is double-grounded if there are disjoint closed Jordan curves $\gamma_{1}$ and $\gamma_{2}$ such that each curve $\alpha \in C$ has one endpoint on $\gamma_{1}$ and the other endpoint on $\gamma_{2}$, and the rest of $\alpha$ is disjoint from both $\gamma_{1}$ and $\gamma_{2}$.

A collection $C$ of $n$ grounded curves can naturally be labeled cyclically along a ground $\gamma$. Start by assigning the label 0 to any curve in $C$ and then proceed to label the remaining curves clockwise, breaking ties arbitrarily, so that the $(i+1)$-th curve along $\gamma$ has label $i$. The labels are elements of $\mathbb{Z}_{n}$. We can define the distance between a pair of grounded curves in $C$ as the cyclic distance between the labels, that is, the distance $d(i, j)$ between the curve labeled $i$ and the one labeled $j$ is $\min (|i-j|, n-|i-j|)$. We let $[i, j]$ denote the cyclic interval of elements $\{i, i+1, \ldots, j\}$.

Lemma 3.1. Suppose $k$ and $d$ are positive integers with $d \geq 6, A$ is a collection of doublegrounded curves with grounds $\gamma_{1}$ and $\gamma_{2}$, and $B$ is a collection of curves such that each curve in $B$ intersects $\gamma_{1}$ and $\gamma_{2}$ in at most $k$ points. If 
(i) each curve in $A$ intersects at most $d$ other curves in $A$; and

(ii) each curve of $B$ intersects at least $15 k d$ curves in $A$,

then there are subsets $A^{\prime} \subset A$ and $B^{\prime} \subset B$ of size $\left|A^{\prime}\right| \geq d / 3$ and $\left|B^{\prime}\right| \geq \frac{d|B|}{3|A|}$ such that every curve in $A^{\prime}$ intersects every curve in $B^{\prime}$.

Proof. We may assume without loss of generality that the Jordan regions bounded by $\gamma_{1}$ and $\gamma_{2}$ are disjoint. Define the cyclic labeling for $A$ based on their endpoints along $\gamma_{1}$. Consider two intersecting curves $\alpha_{i}, \alpha_{j} \in A$ labeled $i$ and $j$, respectively. The endpoints of $\alpha_{i}$ and $\alpha_{j}$ partition $\gamma_{1}$ into two simple curves $\gamma_{1}[i j]$ and $\gamma_{1}[j i]$. One of $\gamma_{1}[i j]$ and $\gamma_{1}[j i]$ together with portions of $\alpha_{i}$ and $\alpha_{j}$ forms a closed Jordan curve $\hat{\gamma}_{1}$ such that the Jordan region bounded by $\hat{\gamma}_{1}$ contains $\gamma_{1}$ and is disjoint from $\gamma_{2}$. Hence, every curve of $A$ whose endpoint lies in the interior of $\hat{\gamma}_{1}$ must intersect $\alpha_{i}$ or $\alpha_{j}$. It follows that if the cyclic distance of two intersecting curves in $A$ is at least $2 d$, then one of them must intersect at least $d$ curves of $A$. Since we assume that no curve in $A$ intersects more than $d$ other curves of $A$, the cyclic distance between any two intersecting curves in $A$ is at most $2 d$.

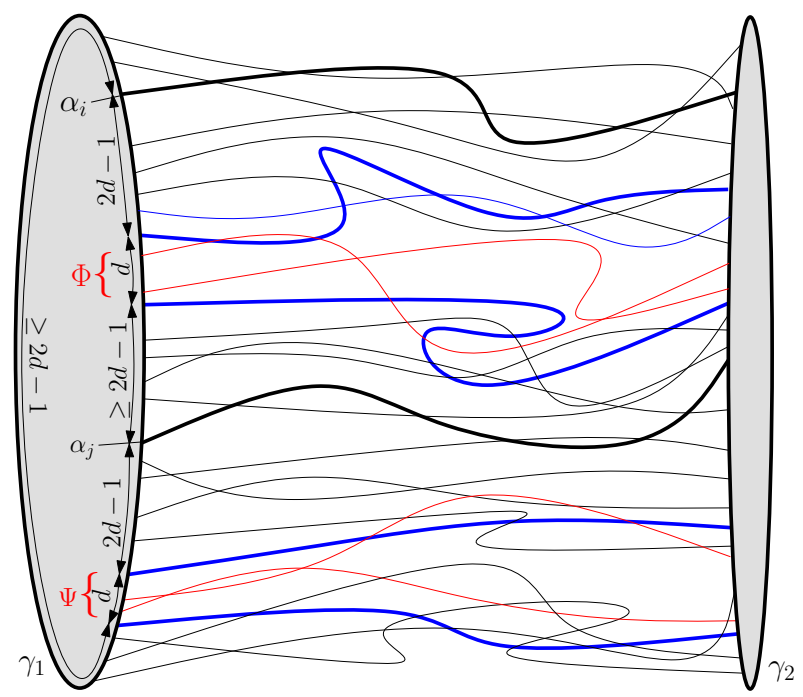

Figure 1. A collection $A$ of double-grounded curves with grounds $\gamma_{1}$ and $\gamma_{2}$. Label the curves cyclically by their endpoints along $\gamma_{1}$. If the cyclic distance of two curves $\alpha_{i}, \alpha_{j} \in A$ is at least $5 d-1$, then every curve $\beta \in B$ that intersects both $\alpha_{i}$ and $\alpha_{j}$ must intersect all curves with label in $\Phi$ or all curves with label in $\Psi$.

Split every curve $\beta \in B$, into at most $2 k+1$ pieces such that each piece, with the possible exception of its endpoints, is disjoint from the grounds $\gamma_{1}$ and $\gamma_{2}$ (refer to Fig. 5). For every $\beta \in B$, let $\beta^{\prime}$ be a piece of $\beta$ that intersects the most curves in $A$. So $\beta^{\prime}$ intersects at least $\frac{15 k d}{2 k+1} \geq 5 d$ curves in $A$. Let $\alpha_{i}$ and $\alpha_{j}$ be curves that intersect $\beta^{\prime}$ and have maximum cyclic distance. Since $\beta^{\prime}$ intersects at least $5 d$ curves in $A$, and $|A| \geq 15 k d>10 d$, there are two curves $\alpha_{i}, \alpha_{j} \in A$ that intersect $\beta^{\prime}$ and whose cyclic distance is at least $5 d-1$. There are cyclic intervals $\Phi \subset[i, j]$ and $\Psi \subset[j, i]$, each of cardinality $d$, such that every curve with label in $\Phi$ or $\Psi$ has cyclic distance at least $2 d$ from both $\alpha_{i}$ and $\alpha_{j}$. Indeed, there are at least $(5 d-2)-2(2 d-1)=d$ curves in each of the cyclic intervals $[i, j]$ and $[j, i]$ at distance at least $2 d$ from both $\alpha_{i}$ and $\alpha_{j}$. Every curve with label in $\Phi$ intersects $\beta^{\prime}$ or every curve with label in $\Psi$ intersects $\beta^{\prime}$ (see Fig. 1). 
Partition $\mathbb{Z}_{|A|}=I_{1} \cup \ldots \cup I_{s}$ into cyclic intervals of size at least $d / 3$ and at most $d / 2$. The number $s$ of cyclic intervals is at most $3|A| / d$. For every $j \in[s]$, let $A_{j}$ denote the set of curves in $A$ whose labels are in $I_{j}$. Since every $\beta \in B$ intersects a set of curves in $A$ whose labels belong to a cyclic interval of size $d$, for each $\beta \in B$, there is an index $j \in[s]$ such that $\beta$ intersects every curve in $A_{j}$. By the pigeonhole principle, there is an index $j \in[s]$ and a subset $B^{\prime} \subset B$ with $\left|B^{\prime}\right| \geq \frac{|B|}{s} \geq \frac{d|B|}{3|A|}$ such that every curve in $B^{\prime}$ intersects every curve in $A_{j}$, Letting $A^{\prime}=A_{j}$, our proof is complete.

The following lemma is used multiple times in the proof of Theorem 1.1.

Lemma 3.2. Let $k \geq 1$ and $m \geq 144$ be integers, let $A$ be a collection of double-grounded curves with grounds $\gamma_{1}$ and $\gamma_{2}$, and let $B$ be a collection of curves such that every curve in $B$ intersects $\gamma_{1}$ and $\gamma_{2}$ in at most $k$ points. If

(i) there are at most $m$ intersecting pairs in $A$, and

(ii) there are at least $20 k \sqrt{m}|B|$ intersecting pairs in $A \times B$,

then there are subsets $A^{\prime} \subset A$ and $B^{\prime} \subset B$ of size $\left|A^{\prime}\right| \geq \sqrt{m} / 6$ and $\left|B^{\prime}\right|>k m|B| /|A|^{2}$ such that every curve in $A^{\prime}$ intersects every curve in $B^{\prime}$.

Proof. Let $A_{1} \subseteq A$ be the set of all curves in $A$ that intersect at most $d=\sqrt{m} / 2$ other curves in $A$. There are at most $2 m / d=4 \sqrt{m}$ curves in $A \backslash A_{1}$, and so there are at most $4 \sqrt{m}|B|$ intersecting pairs in $\left(A \backslash A_{1}\right) \times B$. There are still at least $16 k \sqrt{m}|B|$ intersecting pairs in $A_{1} \times B$. Let $B_{1} \subseteq B$ be the set of curves in $B$ that intersect at least $8 k \sqrt{m}$ curves in $A_{1}$. There are at least $8 k \sqrt{m}|B| /\left|A_{1}\right| \geq 8 k \sqrt{m}|B| /|A|$ elements in $B_{1}$. Applying Lemma 3.1 to $A_{1}$ and $B_{1}$, there are subsets $A^{\prime} \subset A_{1}$ and $B^{\prime} \subset B_{1}$ of size $\left|A^{\prime}\right| \geq d / 3=\sqrt{m} / 6$ and $\left|B^{\prime}\right| \geq d\left|B_{1}\right| /\left(3\left|A_{1}\right|\right)>k m|B| /|A|^{2}$ such that every curve in $A^{\prime}$ intersects every curve in $B^{\prime}$.

\section{Proof of Theorem 1.1 for $[0,1]$-Curves}

Any collection of $[0,1]$-curves between the lines $L_{1}: x=0$ and $L_{1}: x=1$ can also be regarded as a collection of double-grounded curves, where $L_{1}$ and $L_{2}$ act as the grounds. In this section, we establish Theorem 1.1 for $[0,1]$-curves. This special case is the heart of the proof. It will be assumed throughout without loss of generality that the elements of a collection of $[0,1]$-curves have distinct endpoints.

TheOrem 4.1. Given a $k$-intersecting collection $C$ of $n[0,1]$-curves with at least $\varepsilon n^{2}$ intersecting pairs, its intersection graph contains a bi-clique of size at least $c_{k} \varepsilon^{2} n$, where $c_{k}>0$ depends only on $k$.

Roughly speaking, our strategy is to try to prove Theorem 4.1 by induction on $k$. The base case $k=1$ is resolved in Lemma 4.2 below. Ideally, for $k \geq 2$, we would like to find a curve $\Gamma \in C$ such that the portions of the $[0,1]$-curves that intersect $\Gamma$ and stretch between $L_{1}$ and $\Gamma$ form a $(k-1)$-intersecting family $C^{\prime}$ of at least $\Omega_{k}\left(\varepsilon^{2} n\right)$ curves with $\Omega_{k}\left(\left|C^{\prime}\right|^{2}\right)$ intersecting pairs. If this were the case, we could use induction on $k$, because $C^{\prime}$ is homeomorphic to a collection of $[0,1]$-curves where $\Gamma$ acts as the second line $L_{2}$. Unfortunately, we will not be able to exclude that a small number of pairs of curves in $C^{\prime}$ intersect in more than $k-1$ points. This motivates the following definition.

For $\varepsilon, \delta \in(0,1]$ and $k, n \in \mathbb{N}$ with $k \geq 1, n \geq 2$, let $f(\varepsilon, \delta, k, n)$ denote the largest integer $t \in \mathbf{N}$ with the following property: For any collection $C$ of $n[0,1]$-curves in the plane with at least $\varepsilon n^{2}$ intersecting pairs, at most $\delta n^{2}$ pairs of which intersect in more than $k$ points, the intersection graph of $C$ contains $K_{t, t}$. 
Lemma 4.2. For every $\varepsilon \in(0,1]$ and for any integer $n>1$, we have

$$
f\left(\varepsilon, \frac{\varepsilon}{2}, 1, n\right) \geq \frac{\varepsilon}{4} n \text {. }
$$

Proof. Let $C$ be a collection of $n[0,1]$-curves in the plane with at least $\varepsilon n^{2}$ intersecting pairs, at most $\frac{\varepsilon}{2} n^{2}$ of which intersect in more than 1 point. If two curves intersect in exactly one point, then they either properly cross or are tangent to each other. For any curve $\gamma \in C$, let $T(\gamma) \subset C$ consist of all elements of $C$ that intersect $\gamma$ in exactly one point and lie above $\gamma$. Any pair of curves in $T(\gamma)$ have a nonempty intersection. Indeed, if two such curves were disjoint, then one would separate $\gamma$ from the other (see Fig. 2).

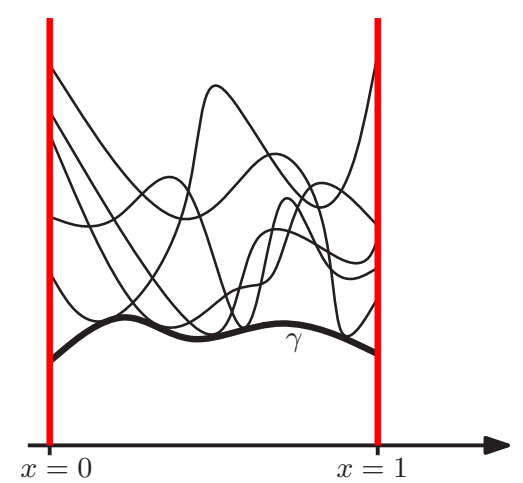

Figure 2. A $[0,1]$-curve $\gamma$, tangent to five other curves lying above it.

There are at least $\frac{\varepsilon}{2} n^{2}$ pairs of curves in $C$ that intersect in exactly one point. If at least $\frac{\varepsilon}{4} n^{2}$ of these pairs are tangent, then there is a curve $\gamma_{0} \in C$ such that $\left|T\left(\gamma_{0}\right)\right| \geq \frac{\varepsilon}{4} n$. Thus, the intersection graph of $C$ contains a clique of size $\frac{\varepsilon}{4} n$, and we are done.

Assume that there are at least $\frac{\varepsilon}{4} n^{2}$ crossing pairs that intersect in exactly one point. Define a two-dimensional partial order $\prec$ on $C$ so that $\alpha \prec \beta$ if the endpoint of $\alpha$ lies below the endpoint of $\beta$ on $L_{i}, i=1,2$. Then the incomparability graph of $(C, \prec)$, which is a subgraph of the intersection graph of $C$, has at least $\frac{\varepsilon}{4} n^{2}$ edges. By Lemma 2.2, the incomparability graph of $(C, \prec)$ (and, hence also the intersection graph of $C$ ) contains $K_{t, t}$ with $t \geq \frac{\varepsilon}{4} n$.

Applying our next theorem successively $k-1$ times, with $t=(10 k)^{-100 k} \varepsilon^{2} n$, we obtain that

$$
f(\varepsilon, 0, k, n) \geq(10 k)^{-100 k} \varepsilon^{2} n,
$$

which proves Theorem 4.1 .

THEOREM 4.3. For all $\varepsilon>0, \delta>0, k \geq 2, n>1$, and $1 \leq t \leq \frac{\varepsilon^{2} n}{10^{7} k}$, we have

$$
f(\varepsilon, \delta, k, n) \geq \min \left(t, f\left(\varepsilon^{\prime}, \delta^{\prime}, k-1, n^{\prime}\right)\right),
$$

where $\varepsilon^{\prime}=\frac{1}{10^{6} k^{2}}, \delta^{\prime}=10^{6}\left(\frac{\delta}{\varepsilon^{4}}+\frac{t}{\varepsilon^{2} n}\right)$, and $n^{\prime}=\left\lceil\frac{\varepsilon^{2} n}{200}\right\rceil$.

Before establishing Theorem 4.3, we make some useful observations. 

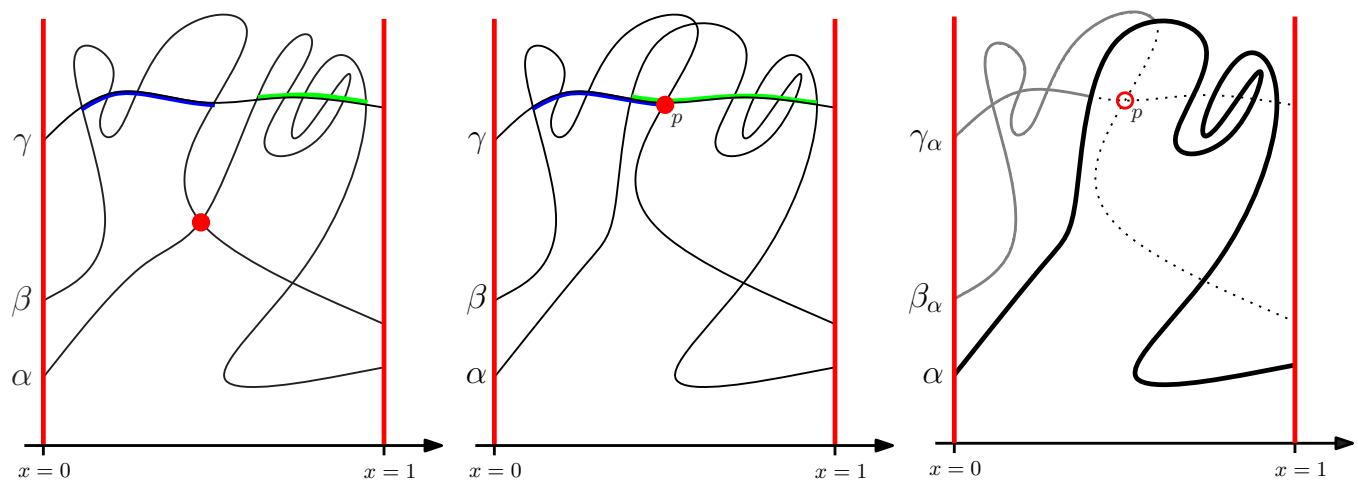

Figure 3. Three $[0,1]$-curves, $\alpha, \beta$, and $\gamma$, with the property that the endpoint of $\gamma$ along $L_{i}$ lies above the corresponding endpoints of $\alpha$ and $\beta(i=1,2)$; and $\gamma$ intersects both $\alpha$ and $\beta$. On the left: If the intersection points $\alpha \cap \gamma$ and $\beta \cap \gamma$ span disjoint subcurves of $\gamma$, then $\alpha$ and $\beta$ must intersect. In the middle: If the intersection points $\alpha \cap \gamma$ and $\beta \cap \gamma$ span overlapping subcurves of $\gamma$, then there is a point $p \in \beta \cap \gamma$ contained in both subcurves of $\gamma$. On the right: If $\alpha$ and $\beta$ intersect, then the portions of $\beta_{\gamma} \subset \alpha$ and $\gamma_{\alpha} \subset \gamma$ between the line $L_{1}$ and their first intersections with $\alpha$ do not contain point $p$.

Observation 1. (See Fig. 3. left side.) Suppose that $\alpha, \beta$, and $\gamma$ are $[0,1]$-curves such that

(i) for $i=1,2$, the endpoints of $\alpha$ and $\beta$ on line $L_{i}$ lie below the endpoint of $\gamma$;

(ii) $\gamma$ intersects both $\alpha$ and $\beta$; and

(iii) the intersection points of $\alpha \cap \gamma$ and $\beta \cap \gamma$ lie in disjoint subcurves of $\gamma$.

Then $\alpha$ and $\beta$ must intersect.

Proof of Theorem 4.3: Let $C$ be a collection of $n[0,1]$-curves with $m \geq \varepsilon n^{2}$ intersecting pairs, at most $\delta n^{2}$ of which intersect in more than $k$ points. Suppose further that $G$, the intersection of graph of $C$, is $K_{t, t}$-free. By Lemma 2.4, $G$ has an induced subgraph $G_{1}$ with $n_{1} \geq \frac{m^{2}}{5 n^{3}} \geq \frac{\varepsilon^{2} n}{5}$ vertices and edge density at least $1-10 t \frac{n^{3}}{m^{2}} \geq 1-\frac{10 t}{\varepsilon^{2} n}$. Let $C_{1} \subseteq C$ denote the collection of curves that correspond to the vertices of $G_{1}$.

Define a linear order $\prec_{1}$ on $C_{1}$ such that $\alpha \prec_{1} \beta$ if the endpoint of $\alpha$ on the line $L_{1}: x=0$ lies above the corresponding endpoint of $\beta$. Also define the 2-dimensional partial order $\prec_{2}$ on $C_{1}$ where $\alpha \prec_{2} \beta$ if and only if $\alpha \prec_{1} \beta$ and the endpoint of $\alpha$ on the line $L_{2}: x=1$ lies above the corresponding endpoint of $\beta$.

For every curve $\gamma \in C_{1}$, let $C_{1}(\gamma)$ be the set of all curves $\alpha \in C_{1}$ with $\alpha \prec_{1} \gamma$ and $\alpha \cap \gamma \neq \emptyset$, and let $d(\gamma)=\left|C_{1}(\gamma)\right|$. For two intersecting curves $\alpha, \beta \in C_{1}$, let $\alpha_{\beta}$ denote the portion of $\alpha$ between its endpoint on $L_{1}$ and its first intersection point with $\beta$. Let $T$ be the set of triples

$$
T=\left\{(\alpha, \beta, \gamma) \in C_{1} \times C_{1} \times C_{1}: \alpha, \beta \in C_{1}(\gamma), \alpha \neq \beta \text {, and } \gamma_{\alpha} \cap \beta \neq \emptyset\right\} .
$$

We give a lower bound on the cardinality of $T$. For any two curves $\alpha, \beta \in C_{1}(\gamma)$, if we follow $\gamma$ from its endpoint at $L_{1}$, we either meet $\alpha$ before $\beta$ or vice versa: We have either $\gamma_{\alpha} \cap \beta \neq \emptyset$ or $\gamma_{\beta} \cap \alpha \neq \emptyset$. Therefore, for each $\gamma$, there are at least $\left(\begin{array}{c}d(\gamma) \\ 2\end{array}\right)$ triples in $T$ with last coordinate $\gamma$, so $|T| \geq \sum_{\gamma \in C_{1}}\left(\begin{array}{c}d(\gamma) \\ 2\end{array}\right)$. Notice that $\sum_{\gamma \in C_{1}} d(\gamma)$ is the number of edges of $G_{1}$. Since the edge density of $G_{1}$ is at least .99, we have $\sum_{\gamma \in C_{1}} d(\gamma) \geq .49 n_{1}^{2}$. By Jensen's inequality, $|T| \geq$ $\sum_{\gamma \in C_{1}}\left(\begin{array}{c}d(\gamma) \\ 2\end{array}\right) \geq \frac{1}{10} n_{1}^{3}$.

A triple $(\alpha, \beta, \gamma) \in T$ is called bad if $\alpha \cap \beta \neq \emptyset$ and $\beta \cap \gamma=\beta_{\alpha} \cap \gamma_{\alpha}$, that is, if all the intersection points of $\beta$ and $\gamma$ are between their portions stretching between $L_{1}$ and $\alpha$. All other triples of $T$ are good. We next give an upper bound on the number of bad triples. Distinguish two kinds of bad triples: A bad triple of the first kind contains at least one pair 
of curves incomparable under $\prec_{2}$; in every remaining bad triple $(\alpha, \beta, \gamma)$, of the second kind, all pairs are pairwise comparable under $\prec_{2}$ but the intersection points $\alpha \cap \gamma$ and $\beta \cap \gamma$ span disjoint subcurves along $\gamma$.

We first show that there are at most $4 t n_{1}^{2}$ bad triples of the first kind: Applying Lemma 2.2 on the 2-dimensional poset $\left(C_{1}, \prec_{2}\right)$, it follows that it contains fewer than $2 t n_{1}$ incomparable pairs. Every incomparable pair can participate in up to $2 n_{1}$ ordered triples $(\alpha, \beta, \gamma)$ with at least one incomparable pair. So there are at most $4 t n_{1}^{2}$ bad triples of the first kind.

Next, we consider bad triples of the second kind. For every curve $\gamma \in C_{1}$, let $C_{2}(\gamma)$ be the set of all curves $\alpha \in C_{1}$ with $\alpha \prec_{2} \gamma$ and $\alpha \cap \gamma \neq \emptyset$. Every curve $\alpha \in C_{2}(\gamma)$ determines a subcurve $\gamma[\alpha]$ of $\gamma$ spanned by the intersection points $\alpha \cap \gamma$. By Observation 1, if two curves $\alpha, \beta \in C_{2}(\gamma)$ determine disjoint subcurves $\gamma[\alpha], \gamma[\beta] \subset \gamma$, then $\alpha \cap \beta \neq \emptyset$. Therefore, the intersection graph $G$ contains the comparability graph of the interval order on $C_{2}(\gamma)$, where $\alpha$ precedes $\beta$ in the interval order if $\gamma[\alpha]$ and $\gamma[\beta]$ are disjoint and $\gamma[\alpha]$ lies closer to $L_{1}$ than $\gamma[\beta]$ along $\gamma$. Since $G$ is $K_{t, t}$-free, for each $\gamma \in C_{1}$, the number of pairs $\alpha, \beta \in C_{2}(\gamma)$ where $\gamma[\alpha]$ is disjoint from $\gamma[\beta]$ is at most $2(t-1)\left|C_{2}(\gamma)\right|<2 t n_{1}$ by Lemma 2.3. Therefore, there are at most $2 t n_{1}^{2}$ triples of the second kind. The total number of bad triples is at most $6 t n_{1}^{2}$.

There are at least $n_{1}^{3} / 10$ triples in $T$, but at most $6 \operatorname{tn}_{1}^{2}$ bad triples. Let $D \subset C_{1}$ be the set where $\alpha \in D$ if there are at least $60 t n_{1}$ bad triples with first coordinate $\alpha$. The size of $D$ is at most $n_{1} / 10$, so there are at most $\frac{n_{1}}{10}\left(\begin{array}{c}n_{1} \\ 2\end{array}\right)<n_{1}^{3} / 20$ triples in $T$ with first coordinate in $D$. Therefore, there are at least $n_{1}^{3} / 20$ triples in $T$ with first coordinate in $C_{1} \backslash D$. Hence, there is $\hat{\alpha} \in C_{1} \backslash D$ such that at least $n_{1}^{2} / 20$ triples in $T$ have first coordinate $\hat{\alpha}$ and at most $60 t n_{1}$ bad triples have first coordinate $\hat{\alpha}$.

Let

$$
\begin{gathered}
A=\left\{\gamma_{\hat{\alpha}}: \hat{\alpha} \in C_{1}(\gamma)\right\} \quad \text { and } \\
B=\left\{\gamma: \gamma \in C_{1}, \gamma \neq \hat{\alpha} \text {, and } \hat{\alpha} \notin C_{1}(\gamma)\right\} \cup\left\{\gamma \backslash \gamma_{\hat{\alpha}}: \hat{\alpha} \in C_{1}(\gamma)\right\} .
\end{gathered}
$$

That is, $A$ is a collection of curves that contains, for each curve in $C_{1}$ that intersects $\hat{\alpha}$ and has a left endpoint above $\hat{\alpha}$, the portion between $L_{1}$ and its first intersection with $\hat{\alpha}$. The collection $B$ contains all other curves of $C_{1}$ entirely, and the remaining portions of the curves covered by $A$. Notice that the collection of curves in $A$ is double grounded with the grounds being the line $L_{1}$ and the curve $\hat{\alpha}$. Also notice that the number of intersecting pairs $\left(\gamma_{\hat{\alpha}}, \beta\right) \in A \times(A \cup B)$ is at least $n_{1}^{2} / 20$; and so the cardinality of $A$ is at least $n_{1} / 20$.

Let $\mu=n_{1}^{2} /\left(10^{6} k^{2}\right)$. First assume that the number of intersecting pairs in $A$ is at most $\mu$. In this case, the number of intersecting pairs in $A \times B$ must be at least $n_{1}^{2} / 20-2 \mu$, which is greater than $20 k \sqrt{\mu} n_{1}$. We can apply Lemma 3.2 and find subsets $A^{\prime} \subset A$ and $B^{\prime} \subset B$ such that

$$
\begin{gathered}
\left|A^{\prime}\right| \geq \frac{\sqrt{\mu}}{6}=\frac{1}{6} \sqrt{\frac{n_{1}^{2}}{10^{6} k^{2}}}>\frac{\varepsilon^{2} n}{10^{5} k} \geq 2 t, \quad \text { and } \\
\left|B^{\prime}\right| \geq \frac{k \mu|B|}{|A|^{2}}=k \cdot\left(n_{1}^{2} /\left(10^{6} k^{2}\right)\right) \cdot \frac{|B|}{|A|^{2}} \geq \frac{k\left(n_{1}^{2} /\left(10^{6} k^{2}\right)\right)}{n_{1}}=\frac{n_{1}}{10^{6} k} \geq \frac{\varepsilon^{2} n}{10^{7} k} \geq 2 t,
\end{gathered}
$$

and every curve in $A^{\prime}$ intersects every curve in $B^{\prime}$. Even though a curve of $C_{1}$ may have a portion in $A$ and a disjoint portion in $B$, the intersection graph of $C$ still contains $K_{t, t}$.

Next assume that the number of intersecting pairs in $A$ is larger than $\mu=n_{1}^{2} /\left(10^{6} k^{2}\right) \geq$ $|A|^{2} /\left(10^{6} k^{2}\right)$. The number of pairs in $A$ that intersect in at least $k$ points cannot exceed

$$
\delta n^{2}+60 t n_{1} \leq\left(\frac{25 \delta}{\varepsilon^{4}}+\frac{300 t}{\varepsilon^{2} n}\right) n_{1}^{2} \leq\left(\frac{25 \delta}{\varepsilon^{4}}+\frac{300 t}{\varepsilon^{2} n}\right)(20|A|)^{2} \leq 10^{6}\left(\frac{\delta}{\varepsilon^{4}}+\frac{t}{\varepsilon^{2} n}\right)|A|^{2} .
$$

We conclude that $f(\varepsilon, \delta, k, n) \geq \min \left(t, f\left(\varepsilon^{\prime}, \delta^{\prime}, k-1, n^{\prime}\right)\right)$. 


\section{Proof of Theorem 1.1}

The aim of this section is to prove Theorem 1.1. In order to use our results on [0,1]-curves and on double-grounded curves, we choose one or two curves to act as grounds. By Observation 2, the intersection graph of the portions of the curves between the chosen ground curves can be realized by $[0,1]$-curves.

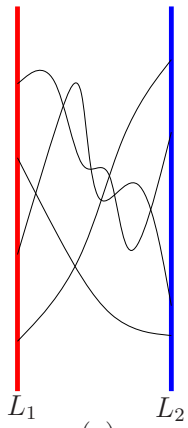

(a)

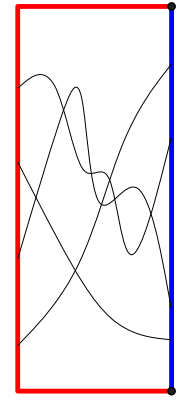

(b)

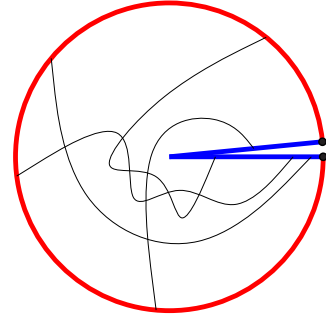

(c)

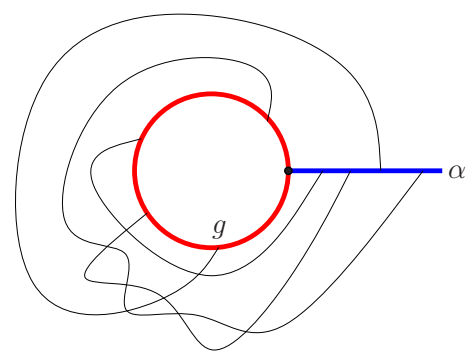

(d)

Figure 4. (a) A collection of [0,1]-curves. (b) We may assume that all curves lie in a rectangle $R$ between vertical lines $L_{1}$ and $L_{2}$. (c) The boundary of $R$ can be continuously deformed to a circular arc and two segments along radii. (d) The segments along the radii can be identified by a further deformation, and an inversion transforms the curves into a collection described in Observation 2, part 2. The above mapping of a collection of curves in (a) to the collection in (d) is invertible.

OBSERVATION 2. The following two statements are equivalent:

(i) $G$ is the intersection graph of a $k$-intersecting collection of $[0,1]$-curves.

(ii) $G$ is the intersection graph of a $k$-intersecting collection $C$ of grounded curves with the following property: We are given a ground $g$ and a curve $\alpha$ with one endpoint incident to $g$ and the rest of $\alpha$ lying in the exterior of $g$. Every curve of $C$ has one endpoint incident to $g$, the other endpoint incident to $\alpha$, and the rest of the curve is disjoint from $\alpha$ and $g$. (See Fig. 4.)

Theorem 5.1. Let $C$ be a $k$-intersecting collection of $n$ curves. Suppose that $C_{1} \subseteq C$ is a collection of grounded curves with ground $g, C_{2}=C \backslash C_{1}$ is a collection of curves disjoint from $g$, and there are at least $\varepsilon n^{2}$ intersecting (nonidentical) pairs in $C_{1} \times C$.

(i) If $C=C_{1}$ (hence $C_{2}=\emptyset$ ), then the intersection graph of $C$ contains a bi-clique of size at least $c_{k} \varepsilon^{8} n$, where $c_{k}>0$ is a constant depending on $k$ only.

(ii) Otherwise, the intersection graph of $C$ contains a bi-clique of size at least $c_{k}^{\prime} \varepsilon^{32} n$, where $c_{k}^{\prime}>0$ is a constant depending on $k$ only.

Proof. For any curve $\gamma \in C_{1}$, let $N(\gamma)$ denote the set of curves in $C$ that intersect $\gamma$, and let $d(\gamma)=|N(\gamma)|$. Label the curves $\alpha \in N(\gamma)$ with integers from 1 to $d(\gamma)$ according to their closest intersection points to the ground along $\gamma$, that is, the label $\ell_{\gamma}(\alpha)$ of $\alpha \in N(\gamma)$ is the number of curves that intersect the portion of $\gamma$ between $g$ and its first intersection with $\alpha$. The sum of labels is $\left(\begin{array}{c}d(\gamma)+1 \\ 2\end{array}\right)$, for every $\gamma \in C_{1}$. Since $\sum_{\gamma \in C_{1}} d(\gamma) \geq \varepsilon n^{2}$, the sum of labels is

$$
\sum_{\gamma \in C_{1}} \sum_{\alpha \in N(\gamma)} \ell_{\gamma}(\alpha)=\sum_{\gamma \in C_{1}}\left(\begin{array}{c}
d(\gamma) \\
2
\end{array}\right) \geq n\left(\begin{array}{c}
\frac{1}{n} \sum_{\gamma \in C_{1}} d(\gamma) \\
2
\end{array}\right)>\frac{\varepsilon^{2}}{4} n^{3}
$$


by Jensen's inequality. Let the weight $w(\alpha)$ of a curve $\alpha \in C$ be the sum of its labels, so $w(\alpha)=\sum_{\gamma: \alpha \in N(\gamma)} \ell_{\gamma}(\alpha)$. Pick a curve $\hat{\alpha}$ of at least average weight, which is at least $\frac{\varepsilon^{2}}{4} n^{2}$.

Let $A$ be the set containing the portion of every curve $\gamma \in N(\hat{\alpha})$ between $g$ and its first intersection with $\hat{\alpha}$. Let $B$ be the set containing all curves $\gamma \in C \backslash N(\hat{\alpha}), \gamma \neq \hat{\alpha}$; and the portion of every curve $\gamma \in N(\hat{\alpha})$ beyond its first intersection with $\hat{\alpha}$. The number of intersecting pairs in $A \times(A \cup B)$ is at least the weight of $\hat{\alpha}$, which is at least $\frac{\varepsilon^{2}}{4} n^{2}$. Since $|B|=n$, we have $|A| \geq \frac{\varepsilon^{2}}{8} n$.

Let $m=\frac{\varepsilon^{4} n^{2}}{10^{4} k^{2}}$. Assume first that the number of intersecting pairs in $A$ is at most $m$. This means that the number of intersecting pairs in $A \times B$ must be at least $\frac{\varepsilon^{2}}{4} n^{2}-2 m$, which is greater than $20 k \sqrt{m}|B|$. By Lemma 3.2, there are subsets $A^{\prime} \subset A$ and $B^{\prime} \subset B$ of size

$$
\begin{gathered}
\left|A^{\prime}\right| \geq \frac{\sqrt{m}}{6}=\frac{1}{6} \sqrt{\frac{\varepsilon^{4} n^{2}}{10^{4} k^{2}}}>\frac{\varepsilon^{2} n}{10^{3} k}, \quad \text { and } \\
\left|B^{\prime}\right| \geq k m|B| /|A|^{2}=k \cdot \frac{\varepsilon^{4} n^{2}}{10^{4} k^{2}} \cdot \frac{|B|}{|A|^{2}} \geq \frac{\varepsilon^{4} n}{10^{4} k}
\end{gathered}
$$

such that every curve in $A^{\prime}$ intersects every curve in $B^{\prime}$. Even though a single curve of $C$ may have a portion in $A$ and a portion in $B$, the intersection graph of $C$ still contains a bi-clique of size at least $c_{k} \varepsilon^{4} n^{2}$ for some constant $c_{k}>0$ depending on $k$.

Assume now that the number of intersecting pairs in $A$ is more than $m$. To prove (i), we assume that $C=C_{1}$ and so $\hat{\alpha} \in C_{1}$. By Observation 2, the intersection graph of $A$ is the intersection graph of $[0,1]$-curves. By Theorem 4.1, the intersection graph of $A$ (and hence, the intersection graph of $C$ ) contains a bi-clique of size at least $c_{k} \varepsilon^{8} n^{2}$ for some constant $c_{k}>0$ depending on $k$. This completes the proof of part (i).

Now we prove (ii). As before, if the number of intersecting pairs in $A$ is at most $m$, then we are done. So we may assume that the number of intersecting pairs in $A$ is larger than $m$. By applying (i) to the collection $A$, it follows that the intersection graph of $A$ (and hence, the intersection graph of $C$ ) contains a bi-clique of size at least

$$
c_{k}\left(\frac{m}{|A|^{2}}\right)^{8}|A|^{2} \geq c_{k} m^{8}|A|^{-14} \geq c_{k}\left(\frac{\varepsilon^{4} n^{2}}{10^{4} k^{2}}\right)^{8} n^{-14} \geq c_{k}^{\prime} \varepsilon^{32} n^{2}
$$

for some constant $c_{k}^{\prime}>0$ depending on $k$.

We are almost ready to prove Theorem 1.1. In the proof, we choose a curve of a $k$-intersecting family as a ground and apply Theorem 5.1(ii). Since each curve intersects any other curve in at most $k$ points, but a ground may intersect each curve in only one point, we keep only a piece of each curve, using the following proposition.
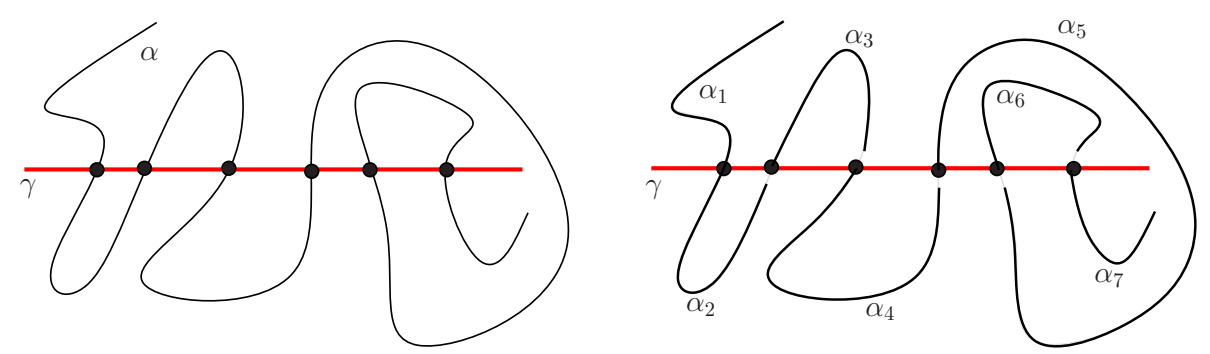

FIGURE 5. If a curve $\alpha$ has $k$ intersection points with $\gamma$, we can split $\alpha$ into $k+1$ disjoint subcurves such that each subcurve intersect $\gamma$ in exactly one point (namely, at an endpoint), and any intersection point with other curves lies in one of the subcurves. 
Proposition 5.2. Let $C$ be a $k$-intersecting collection of $n$ curves, and let $M$ be a set of $m$ intersecting pairs $(\alpha, \beta) \in C \times C$. For every curve $g \in C$, we can choose a subcurve of each curve $\alpha \in C \backslash\{g\}$ such that if $\alpha$ intersects $g$, then $\alpha^{\prime} \cap g$ is an endpoint of $\alpha^{\prime}$, and there are at least $m /(k+1)^{2}$ intersecting pairs $\left(\alpha^{\prime}, \beta^{\prime}\right)$ with $(\alpha, \beta) \in M$.

Proof. Every curve $\alpha \in C$ that intersects $g$ can be split into at most $k+1$ disjoint subcurves, each intersecting $\gamma$ in exactly one point (namely, at an endpoint), see Fig. 5. Let $C^{\prime}$ contain one subcurve of each curve of $C$ chosen uniformly at random. Each intersection point of two curves of $C$ is the intersection point of two subcurves in $C^{\prime}$ with probability at least $1 /(k+1)^{2}$. The expected number of intersecting pairs $\left(\alpha^{\prime}, \beta^{\prime}\right) \in C^{\prime} \times C^{\prime}$ with $(\alpha, \beta) \in M$ is at least $m /(k+1)^{2}$, hence there is a choice of subcurves $C^{\prime}$ that gives at least $m /(k+1)^{2}$ such intersecting pairs.

We now have all the ingredients to prove Theorem 1.1.

Proof of Theorem 1.1: Let $C$ be a $k$-intersecting collection of $n$ curves in the plane with at least $\varepsilon n^{2}$ intersecting pairs. Successively delete every curve of $C$ that intersect fewer than $\frac{\varepsilon}{2} n$ other curves. We are left with a collection $C_{0} \subseteq C$ of curves, each of which intersects at least $\varepsilon n / 2$ other curves of $C_{0}$. Since the deletion of these curves removes fewer than $\varepsilon n^{2} / 2$ intersecting pairs, there are still at least $\varepsilon n^{2} / 2$ intersecting pairs in $C_{0}$.

Choose an arbitrary curve $g \in C_{0}$ and let $C_{1} \subset C_{0}$ be the set of curves that intersect $g$. Since each curve in $C_{0}$ intersects at least $\varepsilon n / 2$ others, there are at least $\varepsilon^{2} n^{2} / 4$ intersecting pairs $(\alpha, \beta) \in C_{1} \times C_{0}$.

By Proposition 5.2, there is a collection $C_{0}^{\prime}$ of subcurves of curves in $C_{0} \backslash\{g\}$ such that the subcurves $C_{1}^{\prime} \subseteq C_{0}^{\prime}$ of the curves of $C_{1}$ are grounded curves with ground $g$ and there are at least $\frac{\varepsilon^{2}}{4} n^{2} /(k+1)^{2}$ intersecting pairs in $C_{1}^{\prime} \times C_{0}^{\prime}$. Applying Theorem 5.1(ii), we conclude that the intersection graph of $C_{0}^{\prime}$, hence that of $C$, contains a bi-clique of size at least $c_{k} \varepsilon^{64} n^{2}$, for some constant $c_{k}>0$ depending only on $k$.

\section{Proof of Theorem 1.3}

We need the following separator theorem from [14], mentioned in the introduction.

LEMma 6.1. [14] The intersection graph of any finite collection of Jordan regions in the plane with a total of $m$ crossings has a separator of size $O(\sqrt{m})$.

Applying this result successively to a collection $C$ of Jordan regions in the plane, we obtain a number of small clusters of $C$ such that any two regions belonging to distinct clusters are disjoint.

THEOREM 6.2. Let $C$ be a collection of Jordan regions with a total of $m$ crossings. For any $d \geq 1$, there exists a partition $C=\bigcup_{i=0}^{s} V_{i}$, for some $s \leq 2 d+1$ such that $\left|V_{0}\right|=O\left((d m)^{1 / 2}\right)$, $\left|V_{i}\right| \leq|C| / d$ for $i=1,2, \ldots, s$, and no element in $V_{i}$ intersects any element in $V_{j}$ for $1 \leq i<$ $j \leq s$.

Proof. The proof is by a simple iteration of Lemma 6.1. At step $i$, we have a collection $S_{i}$ of disjoint subsets of $C$. For a subcollection $W \subseteq C$, let $m(W)$ denote the total number of crossings between the Jordan regions in $W$. Let $q=\left\lceil\log _{3 / 2} d\right\rceil$.

(i) Let $S_{0}=C$ and $i=0$.

(ii) Repeat until $i=q$.

Set $i:=i+1$ and $S_{i+1}:=\emptyset$. For every $W \in S_{i}$, do 
* If $|W| \geq\left(\frac{2}{3}\right)^{i} n$, then apply Theorem 6.1 to partition $W=W_{0} \cup W_{1} \cup W_{2}$ such that $\left|W_{0}\right|=O(\sqrt{m(W)}),\left|W_{1}\right|,\left|W_{2}\right| \leq \frac{2}{3}|W|$, and no set in $W_{1}$ intersects a set in $W_{2}$. In this case, let $S_{i+1}:=S_{i+1} \cup\left\{W_{1}, W_{2}\right\}$.

(iii) Return $S_{q}$.

* Otherwise, let $S_{i+1}=S_{i+1} \cup\{W\}$.

Every $S_{i}$ is a collection of disjoint subsets of $C$. Therefore, we have $\sum_{W \in S_{i}} m(W) \leq$ $2 m$. Since each set partitioned at step $i$ has at least $\left(\frac{2}{3}\right)^{i} n$ elements, the number of subsets partitioned at step $i$ is at most $\left(\frac{3}{2}\right)^{i}$. Thus, for $i \in[q]$, the number of sets in $C$ that belong to one of the subsets in $S_{i}$, but not to any subset in $S_{i+1}$, is

$$
\sum_{W \in S_{i},|W| \geq\left(\frac{2}{3}\right)^{i} n} O(\sqrt{m(W)})=O\left(\left(\frac{3}{2}\right)^{i} \sqrt{\left(\frac{2}{3}\right)^{i} m}\right)=O\left(\left(\frac{3}{2}\right)^{i / 2} \sqrt{m}\right)
$$

by Jensen's inequality, using the concavity of the function $g(x)=\sqrt{x}$. Let $V_{0}$ be the set of elements of $C$ that do not belong to any subsets in $S_{q}$. Summing the number of deleted sets over all steps, we obtain that $\left|V_{0}\right| \leq O\left(\sum_{i=1}^{q}\left(\frac{3}{2}\right)^{i / 2} m^{1 / 2}\right)=O\left((d m)^{1 / 2}\right)$. Denote the elements of $S_{q}$ be $V_{1}, \ldots, V_{s}$, where $s=\left|S_{q}\right|$. In the partition $C=\bigcup_{i=0}^{s} V_{i}$, each set $V_{i} \in S_{q}$ has at most $|C| / d$ elements, and no Jordan region in $V_{i}$ intersects any Jordan region in $V_{j}$, for $1 \leq i<j \leq s$. By grouping together some small $V_{i}$ 's, we may assume that $s \leq 2 d+1$ holds. This completes the proof.

Proof of Theorem 1.3: Let $G$ be the intersection graph of a collection $C$ of $n$ sets belonging to $\mathcal{R}_{k}$. That is, every $\gamma \in C$ can be written as $\gamma=\gamma_{1} \cup \ldots \cup \gamma_{i}$, for some $i \leq k$, where $\gamma_{1}, \ldots, \gamma_{i}$ are Jordan regions from $\mathcal{R}$. The regions $\gamma_{j}$ are called the components of $\gamma$. Let $D$ be the collection of all components of all $\gamma \in C$. Obviously, we have $n \leq|D| \leq k n$.

We distinguish two cases.

Case 1: The intersection graph of $D$ has at most $c n^{2} / k^{3}$ edges, where $c$ is a sufficiently small absolute constant determined by the constant in Theorem 6.2. In this case, applying Theorem 6.2 with $d=3 k^{2}$, there is a partition $D=\bigcup_{i=1}^{s} V_{i}$ with $s \leq 2 d+1,\left|V_{0}\right| \leq n / 3$, and $\left|V_{i}\right| \leq \frac{n}{3 k}$ for $i \in[s]$, such that every set in $V_{i}$ is disjoint from every set in $V_{j}(1 \leq i<j \leq s)$. Let $C_{1}$ be the subset of $C$, none of whose components lie in $V_{0}$, so that $\left|C_{1}\right| \geq 2 n / 3$. Each $\gamma \in C_{1}$ has at most $k$ components, each of which lies in one of the $s$ subsets $V_{1}, \ldots, V_{s}$. Therefore, there are indices $i_{1}, \ldots, i_{k} \in[s]$ and a subcollection $A \subseteq C_{1}$ such that $|A| \geq\left|C_{1}\right| / s^{k}=\Omega\left(n /(7 k)^{2 k}\right)$ and, for each $\gamma \in A$, all of the components of $\gamma$ belong to $V_{i_{1}} \cup \ldots \cup \ldots V_{i_{k}}$. Notice that the number of sets in $C_{1}$ that have at least one component in $V_{i_{1}} \cup \ldots \cup V_{i_{k}}$ is at most $\left|V_{i_{1}} \cup \ldots \cup V_{i_{k}}\right| \leq k|D| / d \leq n / 3$. Therefore, letting $B \subset C_{1}$ be the set of all $\gamma$ whose components all lie in $\cup_{[s] \backslash\left\{i_{1}, \ldots, i_{k}\right\}} V_{i}$, we have $|B| \geq n / 3$ and every set in $B$ is disjoint from every set in $A$.

Case 2: The intersection graph of $D$ has at least $c n^{2} / k^{3}$ edges. In this case, either half of the edges come from containments or at least half of them correspond to boundary intersections. If at least half the intersecting pairs correspond to pairs, for which one of the components of one set contains a component of the other, then one of the components is contained in at least $\frac{c}{2} n / k^{4}$ other components. Then the intersection graph of $C$ contains a clique of size at least $\frac{c}{2} n / k^{5}$. If at least half of the intersecting pairs have components with intersecting boundaries, then we can apply Theorem 1.1 to the boundary curves of the components: The intersection graph of $D$ contains a bi-clique of size $c_{k} n$, where $c_{k}>0$ depends on $k$ only. Since each set of $C$ consists of at most $k$ components, the intersection graph of $C$ contains a bi-clique of size at least $\frac{c_{k}}{k} n$. 


\section{Applications and Open Problems}

First, we mention some applications of Theorem 1.1 and Corollary 1.2. We conclude by listing a few open problems.

In [15], we use Theorem 1.1 to prove the following result. The chromatic number of every $K_{t}$-free intersection graph of $n \geq t$ curves in the plane, with no pair of curves intersecting in more than $k$ points, is at most $\left(c_{k} \frac{\log n}{\log t}\right)^{c \log t}$, where $c>0$ is an absolute constant and $c_{k}>0$ depends only on $k$.

In particular, this implies that, for any $\varepsilon>0$ and for any positive integer $k$, there is a $\delta=\delta(\varepsilon, k)>0$ such that the intersection graph of every $k$-intersecting collection of $n$ curves in the plane contains a clique of size at least $n^{\delta}$ or an independent set of size at least $n^{1-\varepsilon}$. This result is a strengthening of the Erdős-Hajnal property for intersection graphs of $k$-intersecting families of curves. This stronger version was not known even for intersection graphs of straight line segments.

The well-known crossing lemma, proved independently by Ajtai et al. [1] and Leighton [16], states that every graph of $n$ vertices and $m \geq 4 n$ edges, drawn in the plane so that the vertices are represented by points and the edges by continuous arcs connecting the corresponding points, determines $\Omega\left(\mathrm{m}^{3} / \mathrm{n}^{2}\right)$ crossings. This is easily seen to be equivalent to saying that there is always an edge that participates in $\Omega\left(\mathrm{m}^{2} / \mathrm{n}^{2}\right)$ points. In [13], we use Theorem 1.1 to prove a strengthening of the crossing lemma under the assumption that the edges form a $k$-intersecting collection of curves, for some constant $k$. We prove that, for any drawing of a graph $G=(V, E)$ with $n$ vertices and $m \geq 4 n$ edges, with no pair of edges intersecting in more than $k$ points, there are disjoint subsets $E_{1}, E_{2} \subset E$, each of size $\Omega_{k}\left(m^{2} / n^{2}\right)$, such that every edge in $E_{1}$ crosses every edge in $E_{2}$.

Our arguments made essential use of the separator theorem for intersection graphs of families of curves (or Jordan regions) such that the total number of intersections between them is relatively small (see Lemma 6.1). In particular, we used the fact that, for any fixed $k$, the intersection graph $G$ of any $k$-intersecting collection of $n$ curves in the plane has a separator of size $O_{k}(\sqrt{m})$, where $m=|E(G)|$. We do not know whether this statement remains true if we drop the assumption that any pair of curves can intersect in at most a constant number of points. In the literature, intersection graphs of continuous curves in the plane are usually called string graphs.

Question 1. Does every string graph with $m$ edges have a separator of size $O(\sqrt{m})$ ?

It is shown in [15] that the answer is yes for "outerstring graphs," that is, for intersection graphs of grounded curves.

Corollary 1.2 states that the family of string graphs representable as intersection graphs of curves in the plane, any two of which meet in at most a fixed number of points, has the strong Erdős-Hajnal property, and hence the Erdős-Hajnal property. As we have pointed out in the introduction, the family of string graphs does not have the strong Erdős-Hajnal property. We have been unable to answer the following

QuESTION 2. Does the family of string graphs have the Erdős-Hajnal property?

\section{Acknowledgments}

The authors would like to thank Peter Keevash for helpful discussion that led to the separator theorem for curves. 


\section{References}

1. M. Ajtai, V. Chvátal, M. Newborn, and E. Szemerédi, Crossing-free subgraphs, in Theory and Practice of Combinatorics, vol. 60, Mathematical Studies, North-Holland, Amsterdam, 1982, pp. 9-12.

2. N. Alon, J. Pach, R. Pinchasi, R. Radoičić, and M. Sharir, Crossing patterns of semi-algebraic sets, J. Combin. Theory Ser. A 111 (2) (2005), 310-326.

3. N. Alon, J. Pach, and J. Solymosi, Ramsey-type theorems with forbidden subgraphs, Combinatorica 21 (2001), 155-170.

4. S. Basu, Combinatorial complexity in o-minimal geometry, manuscript, 2006. http://www.arxiv.org/abs/math.CO/0612050

5. M. Chudnovsky and S. Safra, The Erdős-Hajnal conjecture for bull-free graphs, in preparation, 2006.

6. G. Ehrlich, S. Even, and R. E. Tarjan, Intersection graphs of curves in the plane, J. Combin. Theory Ser. B 21 (1) (1976), 8-20.

7. P. Erdős, Some remarks on the theory of graphs, Bulletin of the Amer. Math. Soc. 53 (1947), 292-294.

8. P. Erdős and A. Hajnal, Ramsey-type theorems, Discrete Appl. Math. 25 (1989), 37-52.

9. P. Erdős, A. Hajnal, and J. Pach, Ramsey-type theorem for bipartite graphs, Geombinatorics 10 (2000), 64-68.

10. P. Erdős and G. Szekeres, A combinatorial problem in geometry, Compositio Mathematica 2 (1935), 463470.

11. J. Fox, A bipartite analogue of Dilworth's theorem, Order 23 (2-3) (2006), 197-209.

12. J. Fox, J. Pach, and Cs. D. Tóth, Turán-type results for partial orders and intersection graphs of convex sets, submitted, 2007

13. J. Fox, J. Pach, and Cs. D. Tóth, Strengthening the Crossing Lemma, manuscript, 2007.

14. J. Fox and J. Pach, Separator theorems and Turán-type results for planar intersection graphs, submitted, 2007.

15. J. Fox and J. Pach, Coloring planar intersection graphs and the number of edges of $k$-quasi-planar topological graphs, manuscript, 2007.

16. T. Leighton, New lower bound techniques for VLSI, Math. Systems Theory 17 (1984), 47-70.

17. R. J. Lipton and R. E. Tarjan, A separator theorem for planar graphs, SIAM J. Appl. Math. 36 (2) (1979), $177-189$.

18. J. Pach and J. Solymosi, Crossing patterns of segments, J. Combin. Theory Ser. A 96 (2001), 316-325.

19. J. Pach and G. Tóth, Comment of Fox News, Geombinatorics 15 (2006), 150-154.

20. J. B. Sidney, S. J. Sidney, and J. Urrutia, Circle orders, $n$-gon orders and the crossing number, Order 5 (1) (1988), 1-10.

Jacob Fox

Department of Mathematics

Princeton University

Fine Hall, Washington Road

Princeton, NJ 08544-1000

$U S A$

jacobfox@math.princeton.edu

Csaba D. Tóth

Department of Mathematics

Massachusetts Institute of Technology

77 Massachusetts Avenue

Cambridge, MA 02139-4307

USA

toth@math.mit.edu
János Pach

City College, CUNY and

Courant Institute, NYU

251 Mercer Street

New York, NY 10012

USA

pach@cims.nyu.edu 